\title{
CP violation in non-leptonic $B$ decays
}

\section{K. Keri Vos*}

Theoretische Physik 1, Naturwissenschaftlich-Technische Fakultät, Universität Siegen, D-57068 Siegen, Germany

E-mail: keri.vos@uni-siegen. de

Studies of CP violation are an important part of the flavour physics program at $\mathrm{LHCb}$ and the B factories. In this talk, I will present a selected overview of recent theoretical developments in this field where the theoretical challenge is to control the uncertainties from strong interactions to disentangle between the Standard Model and possible New Physics effects. In particular, I discuss the semileptonic asymmetry $a_{\mathrm{sl}}^{s}$, the CKM angle $\gamma$, and the $B_{d}^{0}$ and $B_{s}^{0}$ mixing phases. A newly found puzzle in the $B \rightarrow \pi K$ system is highlighted. Finally, the recent progress to describe three-body $B$ decays in QCD factorization is discussed.

XIV International Conference on Heavy Quarks and Leptons (HQL2018)

May 27- June 1, 2018

Yamagata Terrsa, Yamagata,Japan

${ }^{*}$ Speaker. 


\section{Introduction}

The study of CP violation has already reached an impressive level of precision thanks to a combined theoretical and experimental effort. Such studies test the Standard Model of particle physics (SM) and might reveal new physics (NP). Within the SM, CP violation is described by the Cabibbo-Kobayashi-Maskawa (CKM) matrix. The main theoretical challenge is to disentangle the effects of new physics and strong interaction effects within the SM. Therefore, in order to fully exploit the high level of precision attainable at Belle-II [1] and the LHCb upgrade [2] a continued interplay between theory and experiment is required.

Non-leptonic $B$ decays are the key players in the study of CP violation. The theoretical analyses of these decays are in general challenging due to hadronic matrix elements of four-quark operators entering the corresponding low-energy effective Hamiltonians. However, the flavour symmetries of strong interactions imply relations between the different non-leptonic decays, thereby allowing the elimination of hadronic amplitudes or their determination from experimental data. In this talk, I focus on the recent theoretical progress in the study of CP violation using flavour symmetries. In particular, I will present newly proposed strategies in which theoretical uncertainties can be controlled using experimental data leading to a theoretical precision that matches the (expected) experimental precision. First, in Sec. 2, we discuss the mixing angles $\phi_{d}$ and $\phi_{s}$ which probe CP violation in neutral $B_{d}^{0}$ and $B_{s}^{0}$ meson mixing, respectively. In Sec. 3, we further discuss the non-leptonic decays, and in particular the determinations of the CKM parameter $\gamma$ and the mixing phases $\phi_{s}$ and $\phi_{d}$. Besides the determination of $\phi_{s}$ from tree decays, we discuss in Sec. 3.4 a recent strategy to extract $\phi_{s}$ from the penguin dominated $B_{s}^{0} \rightarrow K^{-} K^{+}$decay. Then we focus on the $B \rightarrow \pi K$ system where a tension with the experimental data is found. Finally, we end with a brief discussion of the recent developments in three-body decays and some concluding remarks.

\section{CP violation in $B_{q}-\bar{B}_{q}$ mixing}

Thanks to quantum-mechanical oscillations the initial $B_{q}^{0}$ meson evolves into a time-dependent linear combination of the $B_{q}^{0}$ and $\bar{B}_{q}^{0}$ states which can be described by a Schrödinger equation. Solving this equation gives the physical mass eigenstates $H$ and $L$

$$
\left|B_{L}\right\rangle=p\left|B_{q}^{0}\right\rangle+q\left|\bar{B}_{q}^{0}\right\rangle, \quad\left|B_{H}\right\rangle=p\left|B_{q}^{0}\right\rangle-q\left|\bar{B}_{q}^{0}\right\rangle,
$$

with the corresponding masses $M_{H}^{q}, M_{L}^{q}$ and the decay widths $\Gamma_{H}^{q}$ and $\Gamma_{L}^{q}$. The mass difference $\Delta M_{q} \equiv M_{H}^{q}-M_{L}^{q} \sim 2\left|M_{12}^{q}\right|$ is dominated by short-distance contributions such that NP can have a significant impact [3]. The width difference $\Delta \Gamma_{q} \equiv \Gamma_{L}^{q}-\Gamma_{H}^{q} \sim 2 \Gamma_{12}^{q} \cos \phi_{q}$ is governed by tree level contributions and is therefore expected to be rather insensitive to NP contributions [4-6]. Finally, $\mathrm{CP}$ violation in mixing gives rise to the mixing phase

$$
\phi_{q} \equiv \arg \left(-\mathrm{M}_{12}^{\mathrm{q}} / \Gamma_{12}^{\mathrm{q}}\right),
$$

which can be probed using flavour-specific semileptonic decays via [4]

$$
a_{\mathrm{sl}}^{q}=\frac{\Gamma\left(\bar{B}_{q}(t) \rightarrow f\right)-\Gamma\left(B_{q}(t) \rightarrow \bar{f}\right)}{\Gamma\left(\bar{B}_{q}(t) \rightarrow f\right)+\Gamma\left(B_{q}(t) \rightarrow \bar{f}\right)}=\left(\frac{\Delta \Gamma_{q}}{\Delta M_{q}}\right) \tan \phi_{q}
$$




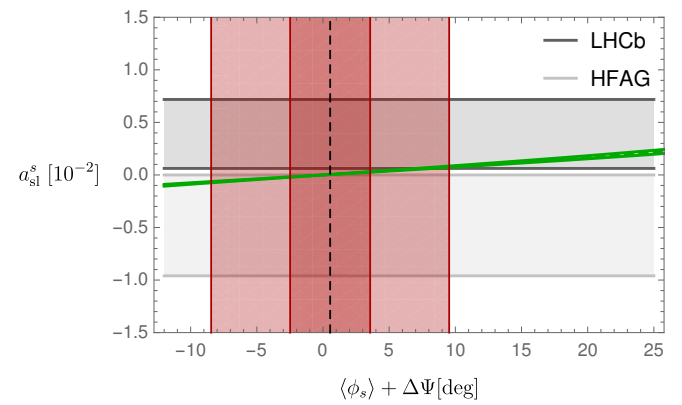

Figure 1: Dependence of $a_{\mathrm{sl}}^{s}$ on $\left\langle\phi_{s}\right\rangle+\Delta \Psi$ from (2.6). The broad vertical bands correspond to the experimental range in (2.8), while the narrow band presents an update scenario. The horizontal bands show the recent LHCb determination [9] and HFAG average [10], respectively.

where the $\bar{B}_{q}(t) \rightarrow f$ and $B_{q}(t) \rightarrow \bar{f}$ transitions can only occur via mixing. In the SM, the flavorspecific CP asymmetries of $B_{d}^{0}$ and $B_{s}^{0}$ can be determined using the heavy-quark expansion and inputs from Lattice QCD [7]:

$$
\left.a_{\mathrm{sl}}^{d}\right|_{\mathrm{SM}}=(-4.7 \pm 0.6) \times 10^{-4},\left.\quad a_{\mathrm{sl}}^{s}\right|_{\mathrm{SM}}=(2.22 \pm 0.27) \times 10^{-5} .
$$

Due to the small SM values, especially for $a_{\mathrm{sl}}^{s}$, any sizeable experimental deviation from this prediction would be a clear sign of new physics. However, such CP-violating NP would also affect the mixing-induced $\mathrm{CP}$ asymmetry of non-leptonic $B$ decays.

\subsection{A closer look at $a_{\mathrm{sl}}^{s}$}

Let us now focus on the $B_{s}-\bar{B}_{s}$ system and the constraints on $a_{\mathrm{sl}}^{s}$ from measurements of CP violation in exclusive $B$ decays [8]. For $\bar{B}_{s}^{0} \rightarrow f$ decays with a final state $f=J / \psi \phi, D_{s}^{-} D_{s}^{+}, J / \psi \pi^{+} \pi^{-}$ caused by $b \rightarrow c \bar{c} s$ processes, measurements of the $\mathrm{CP}$ asymmetries allow the extraction of

$$
\phi_{s}^{f}=\phi_{s}^{\mathrm{SM}}+\phi_{s}^{\mathrm{NP}}+\Delta \psi_{f}^{\mathrm{SM}}+\Delta \psi_{f}^{\mathrm{SM}}
$$

where $\phi_{s}^{\mathrm{SM}}=-(2.1 \pm 0.1)^{\circ}$ [7]. We now rewrite Eq. (2.3) as

$$
a_{\mathrm{sl}}^{s}=\left[(0.46 \pm 0.04) \times 10^{-2}\right] \times \tan \left(\left\langle\phi_{s}\right\rangle+\Delta \Psi\right),
$$

where we inserted the measurements of $\Delta \Gamma_{s}$ and $\Delta M_{s}$. The numerical suppression in Eq. (2.6) already renders the value of $a_{\mathrm{sl}}^{s}$ in the range of the current LHCb measurement [9].

In Eq. (2.6), we used Eq. (2.5) to rewrite $\phi_{s}=\left\langle\phi_{s}\right\rangle+\Delta \Psi$ where $\left\langle\phi_{s}\right\rangle=-(1.5 \pm 1.8)^{\circ}$ is the average of the different available exclusive $\phi_{s}^{f}$ determinations [8]. We emphasize that currently all the determinations of $\phi_{S}$ are consistent with the SM, which significantly constrains possible new physics effects. To quantify this, we introduced

$$
\Delta \Psi=\arg \left[\sum_{\mathrm{f}} \eta_{\mathrm{f}} \mathrm{w}_{\mathrm{f}} \mathrm{e}^{\mathrm{i}\left(\phi_{\mathrm{s}}^{\mathrm{f}}-\left\langle\phi_{\mathrm{s}}\right\rangle\right)}\right], \quad \mathrm{w}_{\mathrm{f}}=\Gamma\left(\mathrm{B}_{\mathrm{s}}^{0} \rightarrow \mathrm{f}\right) \sqrt{\frac{1-\mathrm{A}_{\mathrm{CP}}^{\mathrm{dir}}\left(\mathrm{B}_{\mathrm{s}}^{0} \rightarrow \mathrm{f}\right)}{1+\mathrm{A}_{\mathrm{CP}}^{\mathrm{dir}}\left(\mathrm{B}_{\mathrm{s}}^{0} \rightarrow \mathrm{f}\right)}} .
$$

where $\eta_{f}$ is the $\mathrm{CP}$ eigenvalue of the final state and $w_{f}$ is a measurable weight function.

For the available exclusive measurements we obtain $\Delta \Psi=(2.1 \pm 9.0)^{\circ}$, which yields [8]

$$
a_{\mathrm{sl}}^{s}=(0.004 \pm 0.075) \times 10^{-2} .
$$


In Fig. 1, we show this exclusive constraint combined with the sensitivity of $a_{\mathrm{sl}}^{s}$ on $\left\langle\phi_{s}\right\rangle+\Delta \Psi$ from (2.6) and the experimental measurements of $a_{\mathrm{sl}}^{s}$. We find that our analysis significantly limits the size of $a_{\mathrm{sl}}^{s}$ and thereby also the room for new physics. It will be interesting to confront this picture with more precise measurements, in particular for the $B_{s}^{0} \rightarrow D_{s}^{-} D_{s}^{+}$and $B_{s}^{0} \rightarrow D_{s}^{*-} D_{s}^{*+}$ modes, which dominate the current uncertainty of $\Delta \Psi$. To illustrate the effect of such improved measurements, we have added an experimental benchmark scenario in Fig. 1, in which the uncertainty of $\phi_{s}^{D_{s}^{-} D_{s}^{+}}$is reduced by a factor of three. It would be interesting to confront this picture with more precise data.

\section{CP violation in non-leptonic $B$ decays}

We now focus on CP violation in non-leptonic $B$ decays. The SM low-energy effective Hamiltonian for the $B \rightarrow f$ decay is given by [11]

$$
\left\langle f\left|H_{\mathrm{eff}}\right| B\right\rangle=\frac{G_{F}}{\sqrt{2}} \sum_{j=u, c} V_{j q}^{*} V_{j b}\left(\sum_{i=1,2} C_{i}(\mu)\left\langle f\left|O_{i}^{j q}(\mu)\right| B\right\rangle+\sum_{i=3}^{10} C_{i}(\mu)\left\langle f\left|O_{i}^{q}\right| B\right\rangle\right)
$$

where $V_{i j}$ are the CKM elements and $C_{i}(\mu)$ are the short-distance Wilson coefficients. Longdistance physics is described by the matrix elements of the $O_{i}$ operators. The hadronic matrix elements $\left\langle f\left|O_{i}^{q}\right| B\right\rangle$ can be described in the framework of QCD Factorization (QCDF) [12, 13], where recently, also two-loop contributions have been studied [14]. Despite these efforts power corrections remain difficult to control and in general the description of non-leptonic decays remains a challenge. Alternatively, flavour symmetries can be used to obtain insights into the strong interaction dynamics and its non-perturbative effects. In the following, we focus on strategies that employ flavour symmetries and QCDF to control $S U(3)$ breaking [15].

In the $\mathrm{SM}, \mathrm{CP}$ violation is described by the CKM matrix, which is illustrated by a Unitarity Triangle (UT) with the angles $\alpha, \beta$ and $\gamma$. A variety of non-leptonic flavour observables can be used to determine the UT parameters, exploiting both the direct and mixing-induced CP asymmetries. The direct $\mathrm{CP}$ asymmetry is probed via

$$
A_{\mathrm{CP}}^{\mathrm{dir}}(B \rightarrow f) \equiv \frac{|A(B \rightarrow f)|^{2}-|A(\bar{B} \rightarrow f)|^{2}}{|A(B \rightarrow f)|^{2}+|A(\bar{B} \rightarrow f)|^{2}},
$$

which is governed through the interference between two different decay amplitudes and requires both a non-trivial $\mathrm{CP}$-conserving strong and $\mathrm{CP}$-violating weak phase difference.

For neutral $B_{q}^{0}$ decays, the oscillations give rise to a time-dependent decay rate asymmetry:

$$
A_{\mathrm{CP}}(t) \equiv \frac{\Gamma\left(B_{q}^{0}(t) \rightarrow f\right)-\Gamma\left(\bar{B}_{q}^{0}(t) \rightarrow \bar{f}\right)}{\Gamma\left(B_{q}^{0}(t) \rightarrow f\right)+\Gamma\left(\bar{B}_{q}^{0}(t) \rightarrow \bar{f}\right)}=\frac{A_{\mathrm{CP}}^{\mathrm{dir}} \cos \left(\Delta M_{q} t\right)+A_{\mathrm{CP}}^{\mathrm{mix}} \sin \left(\Delta M_{q} t\right)}{\cosh \left(\Delta \Gamma_{q} t / 2\right)+A^{\Delta \Gamma_{q}} \sinh \left(\Delta \Gamma_{q} t / 2\right)},
$$

where

$$
A_{\mathrm{CP}}^{\mathrm{dir}}\left(B_{q}^{0} \rightarrow f\right) \equiv \frac{1-\left|\lambda_{f}\right|^{2}}{1+\left|\lambda_{f}\right|^{2}}, A^{\Delta \Gamma}\left(B_{q}^{0} \rightarrow f\right) \equiv \frac{-2 \operatorname{Re} \lambda_{\mathrm{f}}}{1+\left|\lambda_{f}\right|^{2}}, \quad \lambda_{f}=\frac{q}{p} \frac{\bar{A}_{f}}{A_{f}},
$$

and which introduces the mixing-induced $\mathrm{CP}$ asymmetry:

$$
A_{\mathrm{CP}}^{\operatorname{mix}}\left(B_{q}^{0} \rightarrow f\right) \equiv \frac{-2 \operatorname{Im} \lambda_{\mathrm{f}}}{1+\left|\lambda_{f}\right|^{2}}=\frac{2\left|\lambda_{f}\right|}{1+\left|\lambda_{f}\right|^{2}} \sin \phi_{q} .
$$




\subsection{Determination of $\gamma$ from $B \rightarrow D K$ decays}

The UT angle $\gamma$ is a key input parameter of the CKM matrix and given by

$$
\gamma=\arg \left(-\frac{\mathrm{V}_{\mathrm{ud}} \mathrm{V}_{\mathrm{ub}}^{*}}{\mathrm{~V}_{\mathrm{cd}} \mathrm{V}_{\mathrm{cb}}^{*}}\right) .
$$

It can be determined using $B^{-} \rightarrow D^{0} K^{-}$and $B^{-} \rightarrow \bar{D}^{0} K^{-}$decays, where the sensitivity to the angle $\gamma$ comes from the interference between the two different decay topologies with $b \rightarrow u \bar{c} s$ and $b \rightarrow c \bar{u} s$ which subsequently decay to the same final state $f$. Since these decays are goverend by tree-level transitions and in particular no penguin operators contribute, the determination of $\gamma$ is theoretically clean. In fact, even electroweak box corrections are tiny [16]. Due to these favourable features, an experimental precision of $1^{\circ}$ is expected to be reachable at Belle-II [1] and the LHCb upgrade [2]. These exciting prospect make the angle $\gamma$ also an interesting external input parameter in the analyses of non-leptonic decays, as discussed in the following subsections.

Finally, also a time-dependent analysis of $B_{s}^{0} \rightarrow D_{s}^{ \pm} K^{\mp}$ (and similar) decays provides a theoretically clean probe of $\gamma+\phi_{s}$ [17-19]. Using the CP asymmetries in $B_{s}^{0} \rightarrow D_{s}^{\mp} K^{ \pm}$system, a measurement of $\gamma$ from the $B_{s}$ system was obtained [20], and it will be interesting to see how this measurement will be improved by the LHCb upgrade.

\subsection{The mixing angles $\phi_{d}$ and $\phi_{s}$}

Measurements of the mixing phases $\phi_{d}$ and $\phi_{s}$ play a key role in testing the SM, as new physics might enter. In the SM

$$
\phi_{d}^{\mathrm{SM}} \equiv 2 \beta=2 \arg \left(-\frac{\mathrm{V}_{\mathrm{cd}} \mathrm{V}_{\mathrm{cb}}^{*}}{\mathrm{~V}_{\mathrm{td}} \mathrm{V}_{\mathrm{tb}}^{*}}\right), \phi_{\mathrm{s}}^{\mathrm{SM}} \equiv 2 \beta_{\mathrm{s}}=2 \arg \left(-\frac{\mathrm{V}_{\mathrm{ts}} \mathrm{V}_{\mathrm{tb}}^{*}}{\mathrm{~V}_{\mathrm{cs}} \mathrm{V}_{\mathrm{cb}}^{*}}\right)
$$

The golden mode for the determination of $\phi_{d}$ is $B_{d} \rightarrow J / \psi K_{S}$ and $B_{s} \rightarrow J / \psi \phi$ for $\phi_{s}$ [21]. Currently these phases are determined at the few degree level and are in agreement with the SM predictions. With Belle-II and the LHC upgrade, we will enter a new era of precision physics and an experimental precision of $O\left(0.5^{\circ}\right)[1,2]$ is expected.

The CP asymmetries in these decays determine the "effective" mixing angle

$$
\sin \phi_{q}^{\mathrm{eff}}=\frac{A_{\mathrm{CP}}^{\mathrm{mix}}\left(B_{q}^{0} \rightarrow f\right)}{\sqrt{1-A_{\mathrm{CP}}^{\mathrm{dir}}\left(B_{q}^{0} \rightarrow f\right)^{2}}}=\sin \left(\phi_{q}^{\mathrm{SM}}+\Delta \phi_{q}+\phi_{q}^{\mathrm{NP}}\right),
$$

where the penguin shift $\Delta \phi_{q}$ is decay mode specific [21]. Although this term is doubly Cabibbo suppressed, these hadronic effect are mandatory to control in order to differentiate between the SM and NP. Using $S U(3)$ symmetry, which provide valuable insights into the hadronic parameters [21-26], these penguin effects can be controlled [27].

\subsection{Controlling penguin effects in $B_{d}^{0} \rightarrow J / \psi K_{S}$ and $B_{s}^{0} \rightarrow J / \psi \phi$}

Assuming only contributions from tree and penguin topologies, we parametrize the golden decay modes $B_{d}^{0} \rightarrow J / \psi K_{S}$ and $B_{s}^{0} \rightarrow J / \psi \phi$ as [22]

$$
A\left(B_{q}^{0} \rightarrow f\right)=\left(1-\frac{\lambda^{2}}{2}\right) \mathscr{C}^{\prime}\left[1+\varepsilon a_{f}^{\prime} e^{i \theta_{f}^{\prime}} e^{i \gamma}\right]
$$




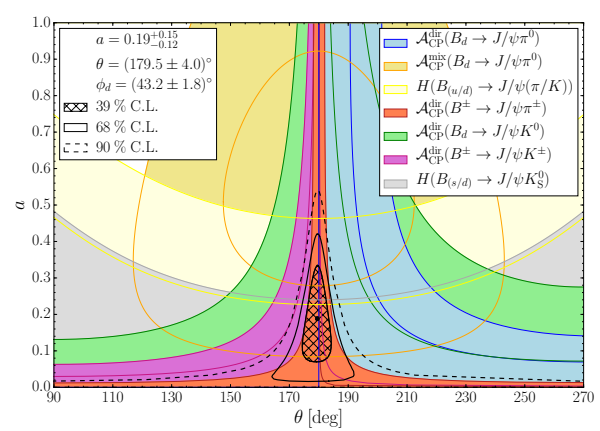

(a)

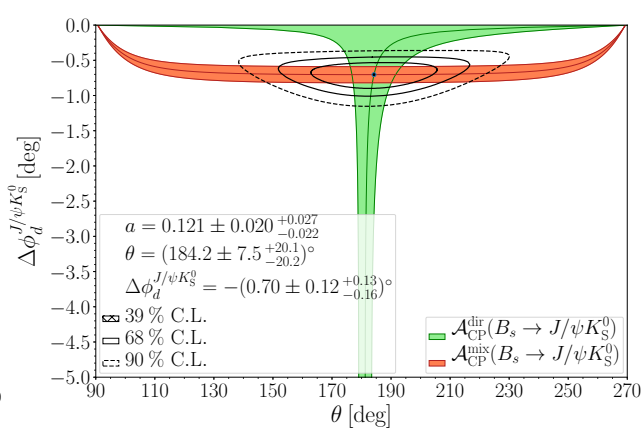

(b)

Figure 2: Determination of $a$ and $\theta$ from current data of $B_{q} \rightarrow J / \psi P$ decays [27] and (b) Scenario to illustrate the determination of $\Delta \phi_{d}$ from the $B_{s}^{0} \rightarrow J / \psi K_{S}$ CP asymmetries (update of [27] by K. de Bruyn).

where $\varepsilon=\frac{\lambda^{2}}{1-\lambda^{2}} \sim 0.05$ and $\lambda=\left|V_{u s}\right|$ is the CKM element. Here $\mathscr{C}^{\prime}$ is a CP-conserving hadronic amplitude, $a^{\prime}$ and the CP-conserving strong phase $\theta^{\prime}$ parametrize the QCD penguin contributions. Using Eq. (3.9), the hadronic phase shift $\Delta \phi_{q}$ and the CP asymmetries can be expressed in terms of the $a^{\prime}$ and $\theta^{\prime}$, which can be determined using $U$-spin partner decays in which the penguin effects are not suppressed. For $B_{d}^{0} \rightarrow J / \psi K_{S}$ the most prominent candidate is the $B_{s}^{0} \rightarrow J / \psi K_{S}$ decay [22]. For the $B_{s}^{0} \rightarrow J / \psi \phi$ decay, suitable penguin control channels are modes with two vector mesons in the final states, most prominently $B_{d}^{0} \rightarrow J / \psi \rho[21,22]$.

In terms of the hadronic parameters, the penguin control decays are written as

$$
A\left(B_{q} \rightarrow f\right)=-\lambda \mathscr{C}\left[1-a_{f} e^{i \theta_{f}} e^{i \gamma}\right]
$$

Using now $\gamma$ as external input, the penguin parameters $a$ and $\theta$ can be extracted in a clean way from measurements of the $\mathrm{CP}$ asymmetries. In the limit of $S U(3)$ symmetry, $a=a^{\prime}$ and $\theta=\theta^{\prime}$. These relations are only affected by non-factorizable $U$-spin breaking corrections [27]. Unfortunately, the $\mathrm{CP}$ asymmetries of the $B_{s}^{0} \rightarrow J / \psi K_{S}$ have not yet been measured. However, the penguin parameters can be determined when combining all measurements for $B_{q} \rightarrow J / \psi P$ decays as shown in Fig. 2a. The extracted penguin parameters result in a penguin shift of (update of [27]):

$$
\Delta \phi_{d}^{J / \psi K_{S}}=\left(-0.71_{-0.65}^{+0.56}\right)^{\circ},
$$

which relies on some theoretical assumptions. In addition, we show in Fig. $2 \mathrm{~b}$ a benchmark scenario for future measurements of the $\mathrm{CP}$ asymmetries in $B_{s}^{0} \rightarrow J / \psi K_{S}$ which gives (update of [27]):

$$
\Delta \phi_{d}^{J / \psi K_{S}}=\left(-0.70 \pm 0.12(\text { stat })_{-0.16}^{+0.13}(\mathrm{U} \text {-spin })\right)^{\circ},
$$

which is at the same level as the expected experimental precision and shows that the penguin shift is controlled by data.

For the $\phi_{s}$ determination, the penguin shift can be determined in a similar way using the CP asymmetries in the $B_{d}^{0} \rightarrow J / \psi \rho^{0}$ control channel. In fact, this strategy is already implemented by $\mathrm{LHCb}$ and their analysis shows that the penguin shifts are tiny and well under control [28]. 


\subsection{Extraction of $\phi_{s}$ from $B_{s}^{0} \rightarrow K^{-} K^{+}$}

It is interesting to compare the determinations of $\gamma$ and $\phi_{s}$ from tree decays to those from penguin dominated decays, as this sector is in particular sensitive to new heavy particles that might enter in the loops [29]. An interesting decay for the extraction of $\gamma$ and $\phi_{s}$ is the QCD penguin dominated $B_{s}^{0} \rightarrow K^{-} K^{+}$decay [15]. Using flavour symmetries, the required hadronic inputs can be related to those of the U-spin partner decay $B_{d}^{0} \rightarrow \pi^{-} \pi^{+}$decay [30-33]. Using the first measurement of CP violation in $B_{s}^{0} \rightarrow K^{-} K^{+}$, the LHCb collaboration determined [34,35]

$$
\gamma=\left(63.5_{-6.7}^{+7.2}\right)^{\circ} \quad \phi_{s}=-\left(6.9_{-8.0}^{+9.2}\right)^{\circ},
$$

which agrees with the determinations from pure tree decays. However, the theoretical precision of this method is limited by $U$-spin breaking corrections to the penguin topologies, making it challenging to reduce the theoretical uncertainty to below $O\left(0.5^{\circ}\right)$ [36]. Therefore, a new strategy was proposed [36] in which both $\gamma$ and $\phi_{d}$ are employed as input parameters such that the $S U(3)$ breaking effects can be probed.

Using the CP asymmetries $B_{s}^{0} \rightarrow K^{-} K^{+}$and $B_{d}^{0} \rightarrow \pi^{-} \pi^{+}$, the hadronic parameter $\Delta \phi_{s}$ in Eq. (3.8) for the $B_{s}^{0} \rightarrow K^{-} K^{+}$decay which we define as $\Delta \phi_{K K}$ is determined. Combined with the determination of $\phi_{s}^{\text {eff }}$ from the $B_{s}^{0} \rightarrow K^{-} K^{+}$CP asymmetries via Eq. (3.8) this then allows for the extraction of $\phi_{s}$. In the determination of $\Delta \phi_{K K}$ both the factorizable and non-factorizable $U$-spin corrections are taken into account. The first are probed using

$$
R_{\pi} \equiv \frac{\Gamma\left(B_{d}^{0} \rightarrow \pi^{-} \pi^{+}\right)}{\left|d \Gamma\left(B_{d}^{0} \rightarrow \pi^{-} \ell^{+} v_{\ell}\right) / d q^{2}\right|_{q^{2}=m_{\pi}^{2}}}, R_{K} \equiv \frac{\Gamma\left(B_{s}^{0} \rightarrow K^{-} K^{+}\right)}{\left|d \Gamma\left(B_{s}^{0} \rightarrow K^{-} \ell^{+} v_{\ell}\right) / d q^{2}\right|_{q^{2}=m_{K}^{2}}} .
$$

In addition, non-factorizable $U$-spin effects are probed by [36]

$$
\xi_{\mathrm{NF}}^{a} \equiv\left|\frac{a_{\mathrm{NF}}}{a_{\mathrm{NF}}^{\prime}}\right|=\left|\frac{a_{\mathrm{NF}}^{T}}{a_{\mathrm{NF}}^{T \prime}}\right|\left|\frac{1+r_{P}}{1+r_{P}^{\prime}}\right|\left|\frac{1+x}{1+x^{\prime}}\right| \text {, }
$$

where the primes indicate a $b \rightarrow s$ transition such that $\xi_{\mathrm{NF}}^{a} \rightarrow 1$ in the $\mathrm{SU}(3)$ limit and $r_{P} \equiv$ $P^{(u t)} / T$ and $x \equiv E+P A^{(u t)} / T+P^{(u t)}$ are ratios of tree (T), penguin (P), exchange (E) and penguinannihilation (PA) topologies.

The parameter $\xi_{\mathrm{NF}}^{a}$ has a theoretically favourable and robust structure and allows the use of data to quantify $U$-spin breaking corrections. We estimate the effect of $\mathrm{SU}(3)$ breaking by allowing for $20 \% U$-spin breaking. We then find, for the tree-level contributions $a_{\mathrm{NF}}^{T}$ which were calculated in QCDF [14] a correction of $O(1 \%)$. The hadronic parameters $r_{P}$ and $x$ can be determined from data and are $O(0.2)$ [36], such that we obtain a correction of $O(4 \%)$ to the ratio $\xi_{\mathrm{NF}}^{a}$. However, the effects of $U$-spin breaking can be probed directly from future data. Specifically, measurements of $\mathrm{CP}$ asymmetries of the pure $\mathrm{P}$ decays $B_{d}^{0} \rightarrow K^{0} \bar{K}^{0}, B_{s}^{0} \rightarrow K^{0} \bar{K}^{0}$, would allow for a determination of both $r_{P}$ and $r_{P}^{\prime}$, while the pure $\mathrm{E}$ and PA decays $B_{d}^{0} \rightarrow K^{+} K^{-}, B_{s}^{0} \rightarrow \pi^{+} \pi^{-}$would probe $x$ and $x^{\prime}$. Finally, combining these corrections leads to $\xi_{\mathrm{NF}}^{a} \sim O(5 \%)$ [36].

Matching the expected experimental precision of $0.5^{\circ}$ in the upgrade era, would require both a $5 \%$ precision on differential rate of $B_{s}^{0} \rightarrow K^{-} \ell^{+} v_{\ell}$ and a $5 \%$ precision on the $S U(3)$-breaking corrections probed by $\xi_{\mathrm{NF}}^{a}$. Unfortunately, measurements of the differential decay rate of $B_{s}^{0} \rightarrow$ $K^{-} \ell^{+} v_{\ell}$ are not available. They are strongly encouraged in order to apply the new strategy. Comparing $\phi_{s}$ obtained from the penguin-dominated $B_{s}^{0} \rightarrow K^{-} K^{+}$decays to the SM predictions and determinations from tree decays as discussed in Sec. 3.2 might reveal new sources of $\mathrm{CP}$ violation. 


\section{Search for new physics in $B \rightarrow \pi K$}

The $B \rightarrow \pi K$ decays are particularly interesting to test the SM, especially to probe possible new physics effects in the electroweak (EW) penguin sector. These decays have been in the spotlight for decades as previous data showed puzzling patterns (see e.g. [37-40]).

The EW penguins are parametrized by

$$
q e^{i \phi} e^{i \omega} \equiv-\left(\hat{P}_{E W}^{\prime}+\hat{P}_{E W}^{\prime \mathrm{C}}\right) /\left(\hat{T}^{\prime}+\hat{C}^{\prime}\right)
$$

where $\phi(\omega)$ is a CP-violating (conserving) phase. The phase $\omega$ is small and vanishes in the $S U(3)$ limit [41]. Here $P_{E W}^{\prime}\left(\hat{T}^{\prime}\right)$ and $P_{E W}^{\prime C}\left(\hat{C}^{\prime}\right)$ denote the colour-allowed and colour-suppressed EW penguin (tree) amplitudes. In the SM, these EW penguin parameters can be calculated using $S U(3)$ flavour symmetry [41-43], yielding $q_{\mathrm{SM}}=(0.68 \pm 0.05) R_{q}$ where $R_{q}$ may differ from 1 through $S U$ (3) breaking corrections. In the SM, $\phi=0$, therefore, a non-zero phase $\phi$ would be a "smokinggun" signal for new CP violating physics. Recently, we pointed out that the current $B_{d}^{0} \rightarrow \pi^{0} K_{S} \mathrm{CP}$ asymmetries are in tension with the SM [44].

The $B_{d}^{0} \rightarrow \pi^{0} K^{0}$ decay is of particular interest, because it is the only $B \rightarrow \pi K$ mode that exhibits a mixing-induced $\mathrm{CP}$ violation. It is given by

$$
A_{\mathrm{CP}}^{\mathrm{mix}}\left(B_{d}^{0} \rightarrow \pi^{0} K^{0}\right) \equiv S_{\mathrm{CP}}^{\pi^{0} K_{S}}=\sin \left(\phi_{d}-\phi_{00}\right) \sqrt{1-\left(A_{\mathrm{CP}}^{\pi^{0} K_{S}}\right)^{2}},
$$

where $\phi_{00}=\arg \left(\overline{\mathrm{A}}_{00} \mathrm{~A}_{00}^{*}\right)$ with $A_{00} \equiv A\left(B_{d}^{0} \rightarrow \pi^{0} K_{S}\right)$ and its CP conjugate decay amplitude $\bar{A}_{00}$. The $B \rightarrow \pi K$ decays obey the isospin amplitude relation $[45,46]$

$$
\sqrt{2} A\left(B_{d}^{0} \rightarrow \pi^{0} K^{0}\right)+A\left(B_{d}^{0} \rightarrow \pi^{-} K^{+}\right)=-\left(\hat{T}^{\prime}+\hat{C}^{\prime}\right)\left(e^{i \gamma}-q e^{i \phi} e^{i \omega}\right)=3 A_{3 / 2} \equiv 3\left|A_{3 / 2}\right| e^{i \phi_{3 / 2}},
$$

and similar for the CP-conjugate decays. This amplitude relation can be used to determine $\phi_{00}$ via amplitude triangles (see [44] for a detailed discussion). Using Eq. (4.2), then gives a theoretically clean correlation between the direct and mixing-induced CP asymmetries in $B_{d}^{0} \rightarrow \pi^{0} K_{S}[39,44$, 47]. This determination only requires additional information on the normalization of the $I=3 / 2$ amplitude, which can be obtained using the $S U(3)$ relation $[42,48]$

$$
\left|\hat{T}^{\prime}+\hat{C}^{\prime}\right|=R_{T+C}\left|\frac{V_{u s}}{V_{u d}}\right| \sqrt{2}\left|A\left(B^{+} \rightarrow \pi^{+} \pi^{0}\right)\right|,
$$

where $R_{T+C}=1.2 \pm 0.2$ and the uncertainty accounts for non-factorizable $S U(3)$ breaking [12, 13]. In addition, we implement a constraint on the triangle amplitude configurations obtained from $B \rightarrow \pi \pi$ data. For current data, we find a tension in the experimental data which might indicate NP in the EW penguin sector [44]. To further study this sector and to reveal the underlying dynamics, it is interesting to determine the electroweak penguin parameters $q$ and $\phi$ using the analogue of the isospin amplitude relation in Eq. (4.3) for the charged $B \rightarrow \pi K$ decays. Using then the measurements of the direct $\mathrm{CP}$ asymmetry and the braching ratio of the $B^{+} \rightarrow \pi^{+} K^{0}$ and $B^{+} \rightarrow \pi^{0} K^{+}$decays, we can determine $q$ as a function of $\phi$ as given in Fig. 3. The different branches arise because the amplitude triangles obtained through Eq. (4.3) have a four-fold ambiguity. We note that there is still a lot of room for NP. 


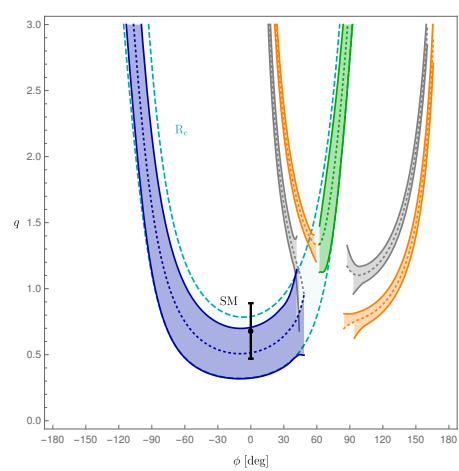

(a)

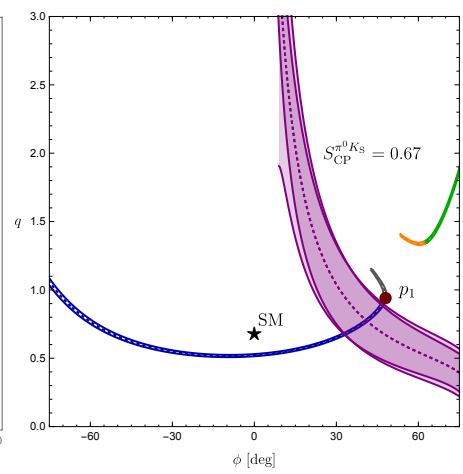

(b)

Figure 3: (a) Contours in the $\phi-q$ plane for the charged $B \rightarrow \pi K$ decays. (b) Future benchmark scenario considering also the mixing-induced CP asymmetry in the $B_{d}^{0} \rightarrow \pi^{0} K_{S}$ decays [44].

In the future, the EW parameters $q$ and $\phi$ can actually be determined using in addition the mixing-induced CP asymmetry of $B_{d}^{0} \rightarrow \pi^{0} K_{S}$. To illustrate this, we consider a future measurement of $S_{\mathrm{CP}}^{\pi^{0} K_{S}}=0.67$. Figure 3 shows that this new strategy can determine the EW penguin parameters in a theoretically clean way. This offers exciting prospects for Belle-II and the LHCb upgrade.

\section{CP violation in three-body decays}

Hadronic three-body decays constitute a large part of the branching fraction for non-leptonic $B$ decays. Due to there non-trivial kinematics, they contain much more information on strong phases than two-body decays. Interesting patterns of $\mathrm{CP}$ violation were found in the experimental data, especially for $B \rightarrow \pi \pi \pi$ decays [49]. The theoretical description of these decays is challenging and they have been studied in a large variety of approaches, with a recent first attempt using QCDF $[50,51]$.

Following [50], the Dalitz plot distribution can be split into three different regions, where different factorization descriptions apply. In the central region, where all the invariant masses are roughly the same, the amplitude is expected to factorize completely. However, the $b$-quark is too light for this complete factorization to occur. Therefore, the complete Dalitz plot consists only of edges (i.e. regions where two of the decaying particles move collinearly). Here all the resonances are located and the decays resemble quasi-two body decays. In this region, full factorization breaks down and new non-perturbative quantities need to be introduced. The factorization theorem in this region is given by [50]

$$
\left\langle\pi^{+} \pi^{+} \pi^{-}\left|O_{i}\right| B\right\rangle=T_{i}^{I} \otimes F^{B \rightarrow \pi^{+}} \otimes \Phi_{\pi^{+} \pi^{-}}+T_{i}^{I I} \otimes F^{B \rightarrow \pi^{+} \pi^{-}} \otimes \Phi_{\pi^{+}},
$$

where $T_{i}$ are perturbatively calculable hard scattering kernels, $\phi_{\pi}$ and $F^{B \rightarrow \pi}$ are the pion lightcone distribution amplitude (LCDA) and the $B \rightarrow \pi$ form factor which are known from two-body decays. The new elements are the $B \rightarrow \pi \pi$ form factor $F^{B \rightarrow \pi \pi}[52,53]$ and the $2 \pi$-LCDA, which introduce non-perturbative strong phases. The $B \rightarrow \pi \pi$ vector form factor was studied using lightcone sum rules [54], while the normalization of the $2 \pi$-LCDA can be obtained from $e^{+} e^{-}$data. 
Using this information, a first study of the leading-order contributions to $\mathrm{CP}$ violation in $B \rightarrow \pi \pi \pi$ using QCDF was performed [51]. However, the lack of knowledge of the scalar $B \rightarrow \pi \pi$ form factor and higher-order corrections currently limits this study. Further investigations are required to clarify these issues. Especially considering the large amount of data that will become available, three-body decays offer interesting avenues that should still be explored.

\section{Summary}

A continued combined effort between theorists and experimentalists is required to fully exploit the upcoming high-precision era in flavour physics. Theoretically, the main challenge is to find the cleanest strategies in which theoretical uncertainties are well under control and can be further reduced through future experimental data. In this talk, I discussed the clean determination of the semileptonic asymmetry $a_{\mathrm{sl}}^{s}$, the UT angle $\gamma$ and the mixing phases $\phi_{s}$ and $\phi_{d}$ using flavour symmetries. The $S U$ (3)-breaking corrections are controlled using QCDF and experimental inputs. This also allows for an extraction of the hadronic parameters, which give insight into long distance physics, thereby paving the road for future theoretical progress. The new strategies offer interesting prospects for Belle-II and the LHCb upgrade, and show that the uncertainties from strong interaction effects can be sufficiently controlled. Finally, we may either once again confirm the SM or establish new physics.

\section{Acknowledgments}

I would like to thank Robert Fleischer for the pleasant collaboration and useful discussions. I would also like to thank Kristof de Bruyn for providing updated plots and numerics for Sec. 3.2, and Ruben Jaarsma, Rebecca Klein, Eleftheria Malami, Thomas Mannel and Javier Virto for the fruitful collaboration on the various topics discussed in this talk. This work is supported by the Deutsche Forschungsgemeinschaft (DFG) within research unit FOR 1873 (QFET).

\section{References}

[1] T. Abe et al. [Belle-II Collaboration], arXiv:1011.0352 [physics.ins-det]; T. Aushev et al., arXiv:1002.5012 [hep-ex].

[2] R. Aaij et al. [LHCb Collaboration], Eur. Phys. J. C 73, 2373 (2013) [arXiv:1208.3355 [hep-ex]].

[3] L. Di Luzio, M. Kirk and A. Lenz, Phys. Rev. D 97, 095035 (2018) [arXiv:1712.06572 [hep-ph]].

[4] I. Dunietz, R. Fleischer and U. Nierste, Phys. Rev. D 63, 114015 (2001) [hep-ph/0012219].

[5] A. Badin, F. Gabbiani and A. A. Petrov, Phys. Lett. B 653, 230 (2007) [arXiv:0707.0294 [hep-ph]].

[6] A. Lenz, U. Nierste, J. Charles, S. Descotes-Genon, H. Lacker, S. Monteil, V. Niess and S. T'Jampens, Phys. Rev. D 86, 033008 (2012) [arXiv:1203.0238 [hep-ph]].

[7] M. Artuso, G. Borissov and A. Lenz, Rev. Mod. Phys. 88,045002 (2016) [arXiv:1511.09466 [hep-ph]].

[8] R. Fleischer and K. K. Vos, Phys. Lett. B 770, 319 (2017) [arXiv:1606.06042 [hep-ph]]. 
[9] R. Aaij et al. [LHCb Collaboration], Phys. Rev. Lett. 117, 061803 (2016) [arXiv:1605.09768 [hep-ex]].

[10] Y. Amhis et al. [Heavy Flavor Averaging Group (HFAG) Collaboration], arXiv:1412.7515 [hep-ex].

[11] G. Buchalla, A. J. Buras and M. E. Lautenbacher, Rev. Mod. Phys. 68, 1125 (1996) [hep-ph/9512380].

[12] M. Beneke, G. Buchalla, M. Neubert and C. T. Sachrajda, Nucl. Phys. B 606, 245 (2001) [hep-ph/0104110].

[13] M. Beneke and M. Neubert, Nucl. Phys. B 675, 333 (2003) [hep-ph/0308039].

[14] M. Beneke, T. Huber and X. Q. Li, Nucl. Phys. B 832, 109 (2010) [arXiv:0911.3655 [hep-ph]]; G. Bell, M. Beneke, T. Huber and X. Q. Li, Phys. Lett. B 750, 348 (2015) [arXiv:1507.03700 [hep-ph]].

[15] R. Fleischer, Phys. Rept. 370, 537 (2002) [hep-ph/0207108].

[16] J. Brod and J. Zupan, JHEP 1401, 051 (2014) [arXiv:1308.5663 [hep-ph]]; J. Brod, Phys. Lett. B 743, 56 (2015) doi:10.1016/j.physletb.2015.02.022 [arXiv:1412.3173 [hep-ph]].

[17] R. Aleksan, I. Dunietz and B. Kayser, Z. Phys. C 54, 653 (1992).

[18] K. De Bruyn, R. Fleischer, R. Knegjens, M. Merk, M. Schiller and N. Tuning, Nucl. Phys. B 868, 351 (2013) [arXiv:1208.6463 [hep-ph]].

[19] R. Fleischer, Nucl. Phys. B 671, 459 (2003) [hep-ph/0304027].

[20] R. Aaij et al. [LHCb Collaboration], JHEP 1803, 059 (2018) [arXiv:1712.07428 [hep-ex]].

[21] S. Faller, M. Jung, R. Fleischer and T. Mannel, Phys. Rev. D 79, 014030 (2009) [arXiv:0809.0842 [hep-ph]].

[22] R. Fleischer, Phys. Rev. D 60 (1999) 073008 [hep-ph/9903540]; Eur. Phys. J. C 10 (1999) 299 [hep-ph/9903455].

[23] S. Faller, R. Fleischer and T. Mannel, Phys. Rev. D 79, 014005 (2009) [arXiv:0810.4248 [hep-ph]].

[24] M. Jung, Phys. Rev. D 86, 053008 (2012) [arXiv:1206.2050 [hep-ph]].

[25] M. Ciuchini, M. Pierini and L. Silvestrini, Phys. Rev. Lett. 95, 221804 (2005) [hep-ph/0507290]; arXiv:1102.0392 [hep-ph].

[26] M. Gronau and D. London, Phys. Rev. Lett. 65, 3381 (1990).

[27] K. De Bruyn and R. Fleischer, JHEP 1503, 145 (2015) [arXiv:1412.6834 [hep-ph]]; see also K. De Bruyn, PoS BEAUTY 2016, 004 (2016).

[28] R. Aaij et al. [LHCb Collaboration], JHEP 1511, 082 (2015) [arXiv:1509.00400 [hep-ex]].

[29] A. J. Buras and J. Girrbach, Rept. Prog. Phys. 77, 086201 (2014) [arXiv:1306.3775 [hep-ph]].

[30] R. Fleischer, Phys. Lett. B 459, 306 (1999) [hep-ph/9903456].

[31] R. Fleischer, Eur. Phys. J. C 52, 267 (2007) [arXiv:0705.1121 [hep-ph]].

[32] R. Fleischer and R. Knegjens, Eur. Phys. J. C 71, 1532 (2011) [arXiv:1011.1096 [hep-ph]].

[33] M. Ciuchini, E. Franco, S. Mishima and L. Silvestrini, JHEP 1210, 029 (2012) [arXiv:1205.4948 [hep-ph]].

[34] R. Aaij et al. [LHCb Collaboration], JHEP 1310, 183 (2013) [arXiv:1308.1428 [hep-ex]]. 
[35] R. Aaij et al. [LHCb Collaboration], Phys. Lett. B 741, 1 (2015) [arXiv:1408.4368 [hep-ex]].

[36] R. Fleischer, R. Jaarsma and K. K. Vos, JHEP 1703, 055 (2017) [arXiv:1612.07342 [hep-ph]]; Phys. Rev. D 94, 113014 (2016) [arXiv:1608.00901 [hep-ph]].

[37] A. J. Buras, R. Fleischer, S. Recksiegel and F. Schwab, Nucl. Phys. B 697, 133 (2004) [hep-ph/0402112].

[38] R. Fleischer, S. Recksiegel and F. Schwab, Eur. Phys. J. C 51, 55 (2007) [hep-ph/0702275 [HEP-PH]].

[39] R. Fleischer, S. Jager, D. Pirjol and J. Zupan, Phys. Rev. D 78, 111501 (2008) [arXiv:0806.2900 [hep-ph]].

[40] N. B. Beaudry, A. Datta, D. London, A. Rashed and J. S. Roux, arXiv:1709.07142 [hep-ph].

[41] M. Neubert and J. L. Rosner, Phys. Rev. Lett. 81, 5076 (1998) [hep-ph/9809311]; Phys. Lett. B 441, 403 (1998) [hep-ph/9808493].

[42] A. J. Buras and R. Fleischer, Eur. Phys. J. C 11, 93 (1999)

[43] R. Fleischer, Phys. Lett. B 365, 399 (1996) [hep-ph/9509204].

[44] R. Fleischer, R. Jaarsma and K. K. Vos, arXiv:1712.02323 [hep-ph]; arXiv:1806.08783 [hep-ph].

[45] Y. Nir and H. R. Quinn, Phys. Rev. Lett. 67, 541 (1991).

[46] M. Gronau, O. F. Hernandez, D. London and J. L. Rosner, Phys. Rev. D 52, 6374 (1995) [hep-ph/9504327].

[47] M. Gronau and J. L. Rosner, Phys. Lett. B 666, 467 (2008) [arXiv:0807.3080 [hep-ph]].

[48] M. Gronau, J. L. Rosner and D. London, Phys. Rev. Lett. 73, 21 (1994) [hep-ph/9404282].

[49] R. Aaij et al. [LHCb Collaboration], Phys. Rev. D 90, 112004 (2014) [arXiv:1408.5373 [hep-ex]], Phys. Rev. Lett. 112 (2014) 011801 [arXiv:1310.4740 [hep-ex]].

[50] S. Kränkl, T. Mannel and J. Virto, Nucl. Phys. B 899, 247 (2015) [arXiv:1505.04111 [hep-ph]].

[51] R. Klein, T. Mannel, J. Virto and K. K. Vos, JHEP 1710, 117 (2017) [arXiv:1708.02047 [hep-ph]].

[52] S. Faller, T. Feldmann, A. Khodjamirian, T. Mannel and D. van Dyk, Phys. Rev. D 89, 014015 (2014) [arXiv:1310.6660 [hep-ph]].

[53] P. Böer, T. Feldmann and D. van Dyk, JHEP 1702, 133 (2017) [arXiv:1608.07127 [hep-ph]]; T. Feldmann, D. Van Dyk and K.K. Vos, arXiv:1807.01924 [hep-ph].

[54] S. Cheng, A. Khodjamirian and J. Virto, JHEP 1705, 157 (2017) [arXiv:1701.01633 [hep-ph]]; Phys. Rev. D 96, 051901 (2017) [arXiv:1709.00173 [hep-ph]]. 